\section{SF018/\#632 MAYLARD INCISION: A TRANSVERSE INCISION FOR COMPLEX GYNECOLOGIC SURGERY}

${ }^{1} M A$ Ferjaoui ${ }^{*},{ }^{1} S$ Khedhri, ${ }^{2} R$ Arfaoui, ${ }^{3} M A$ Hannachi, ${ }^{1} R$ Azri, ${ }^{2} R$ Bouhmida, ${ }^{4} M$ Malek, ${ }^{4} \mathrm{~K}$ Neji. ${ }^{1}$ Tunis maternity center, Department B of Gynecologic Surgery, Tunis, Tunisia; ${ }^{2}$ Tunis military hospital, Department of Gynecology and Obstetrics, Tunis, Tunisia; ${ }^{3}$ Maternity and neonatology center of Tunis, B, Ben Arous, Tunisia; ${ }^{4}$ Tunis Maternity Center, Department B of Gynecologic Surgery, Tunis, Tunisia

\subsection{6/ijgc-2021-IGCS.62}

Introduction Maylard incision is a transverse abdominal incision characterized by the ligation of inferior epigastric vessels and the transection of rectus abdominus muscles. It provides a good surgical exposure. The aims of this video is to detail Maylard incision technique.

Description A transverse skin incision is made four $\mathrm{cm}$ above the symphisis pubis. After dissection of subcutaneous tissus, transversalis fascia is openned. Rectus abdominus muscles are separated from the fascia. Inferior epigastric vessels are located at the external edge of rectus abdominus muscles. They are dissected, ligated and cutted. The rectus abdominus muscles are sectionned using bipolar device. The parietal peritoneum can be openned. After the surgery, the parietal peritoneum is closed using a running suture. Rectus abdominus muscles can be autres but it is not mandatory.

Conclusion Maylard incision provides a good surgical exposure, a cosmetic, reproducible technique with less complications and pain comparing to midline incision. Hands on training are required to perfom this technique.

\section{SF019/\#636 VASCULAR INJURY IN ROBOT-ASSISTED PARA- AORTIC LYMPHADENECTOMY}

J Hernandez*, J Acosta, L Almeciga, S Vieira. Instituto Nacional de Cancerologia, Gynecology Oncology, Bogota, Colombia

\subsection{6/ijgc-2021-IGCS.63}

First robot-assisted para-aortic lymphadenectomy performed in gynecological oncology in our country for surgical staging of cervical cancer in a patient with radiological evidence of pelvic lymph node involvement. Description of the performance of docking for para-aortic lymphadenectomy by robot-assisted surgery and the presentation of a vascular lesion during dissection of the interaortocaval nodes and its management with vascular clip. Although unplanned conversion-to-open is a rare event occurring in less than $1 \%$ of robotic-assisted cases, it is associated with worse outcomes and carries signifcant morbidity and potentially life-threatening consequences. In the setting of massive hemorrhage, timely and efective emergency undocking followed by emergency laparotomy and obtaining vascular control may be lifesaving. In this case we used a vascular clip to control the bleeding and avoid converting to an open procedure. The presence of vascular lesions during robotic-assisted surgery can occur in up to $3 \%$, depending on the series reviewed. Keep calm and recognize the bleeding site accurately to avoid collateral damage. The surgeon should have knowledge about the different techniques that can be used to control major bleeding. Vascular clips applied properly by trained surgeons provide a safe option for vascular control injury in robotic gynecology surgery. Future research should be aimed at finding the best bleeding control technique in robotic surgery.

\section{SF020/\#652 USE OF A VAGINALLY ADAPTED GAMMA PROBE IN THE VIDEOLAPAROSCOPIC SENTINEL LYMPH NODE RESEARCH}

${ }^{1} \mathrm{~A}$ Munhóz*, ${ }^{2} \mathrm{~B}$ Ribeiro, ${ }^{3} \mathrm{~A}$ Tsunoda, ${ }^{2} \mathrm{~L}$ Pedrini. ${ }^{1}$ Hospital Erasto Gaertner, Ginecologic Oncology, Curitiba, Brazil; ${ }^{2}$ Hospital Erasto Gaertner, Surgical Oncology Resident, Curitiba, Brazil; ${ }^{3}$ Hospital Erasto Gaertner, Surgical Oncology, Curitiba, Brazil

\subsection{6/ijgc-2021-IGCS.64}

Introduction The use of videolaparoscopy in the management of gynecological cancer has gained ground due to the possibility of offering quality treatment with benefits in relation to pain and postoperative recovery, mainly. Despite making it possible to conduct sentinel lymph node research, there is still a limitation regarding the use of the laparoscopic gamma probe, due to the high cost and low frequency of use in institutions that normally have the conventional gamma probe used in open surgery. The purpose of this video is to show the possibility of using the conventional gamma-probe vaginally in laparoscopic surgeries.

Description The video shows the challenge that the surgeon often faces when searching for sentinel lymph nodes using patent blue, when the lymphatic pathways and the sentinel are not stained on either or both sides. In this situation, the use of the Technetium-99m injection in the cervix and lymph node research using a gamma-probe protected by a sterile glove introduced vaginally may be an alternative, contributing to the sentinel meeting.

Conclusion The use of the adapted gamma-probe used vaginally can contribute to the investigation of the sentinel lymph node by a minimally invasive route when a laparoscopic probe is not available, especially in cases of early endometrial cancer, in which only the sentinel lymph node biopsy is sufficient for staging, and can be useful to find lymph nodes and avoid systematic lymphadenectomy and its consequences.

\section{SF021/\#830 MINIMALLY INVASIVE SECONDARY CYTOREDUCTIVE SURGERY FOR HEPATO-RENAL RECESS ISOLATED RECURRENCE OF SEROUS ENDOMETRIAL CANCER IN BRCA1 MUTATED PATIENT}

${ }^{1} \mathrm{~V}$ lacobelli; ${ }^{*}{ }^{2} \mathrm{~F}$ Taliente, ${ }^{3} \mathrm{G}$ Scambia, ${ }^{3} \mathrm{~A}$ Fagotti, ${ }^{3} \mathrm{~F}$ Fanfani, ${ }^{4} \mathrm{GF}$ Zannoni, ${ }^{5} \mathrm{~F}$ Giuliante ${ }^{6} V$ Gallotta. 'Università Cattolica del Sacro Cuore, Rome, Italy, Department of Woman and Child Health and Public Health, Woman Health Area, Rome, Italy; ${ }^{2}$ Catholic University, Rome, Italy, General Surgery Department, Agostino Gemelli Irccs University Hospital Foundation, Hepatobiliary Surgery Unit, Foundation 'policlinico Universitario A. Gemelli', Irccs, Rome, Italy; ${ }^{3}$ Università Cattolica del Sacro Cuore, Department of Woman and Child Health and Public Health, Woman Health Area, Fondazione Policlinico Universitario A. Gemelli Irccs, Roma, Italy; ${ }^{4}$ Fondazione Policlinico Universitario A. Gemelli, IRCCS, Gynecopathology and Breast Pathology Unit, Dipartimento Per La Salute Della Donna E Del Bambino E Della Salute Pubblica, Rome, Italy; ${ }^{5}$ Hepatobiliary Surgery Unit, Foundation 'Policlinico Universitario A. Gemelli', IRCCS, Catholic University, Rome, Italy, General Surgery Department, Agostino Gemelli Irccs University Hospital Foundation, Rome, Italy; ${ }^{6}$ Fondazione Policlinico Universitario Agostino Gemelli, IRCCS, Gynecologic Oncology, Rome, Italy

\subsection{6/ijgc-2021-IGCS.65}

Introduction This film shows the surgical management of a young BRCA1 mutated women affected by a recurrence of serous endometrial carcinoma (SEC). This aggressive subtype of endometrial cancer recurs in $30-80 \%$ of cases and shares most of its molecular features with serous ovarian cancer. The role of BRCA mutations in this setting is still not completely understood. The 